\title{
Response from Yu Xiang to "Comment on The complete nucleotide sequence and genome organization of pea streak virus (genus Carlavirus)"
}

\author{
Yu Xiang ${ }^{1}$
}

Received: 12 August 2015/Accepted: 18 August 2015/Published online: 15 September 2015

(C) Her Majesty the Queen in Right of Canada 2015

\section{Dear Editor,}

We submitted the complete genome sequence of pea streak virus (PeSV) (GenBank accession KP828803) to NCBI GenBank on 19-FEB-2015, then we sent a request to the GenBank for immediate release of the PeSV sequence on 27-MAY-2015, and the sequence was released on 28-JUN2015 by NCBI. We submitted the manuscript "The complete nucleotide sequence and genome organization of pea streak virus (genus Carlavirus)" (DOI: 10.1007/s00705015-2467-2) to Archives of Virology (ARVI) on 13-MAR2015. Our manuscript was scientifically accepted on 16-APR-2015, and finally accepted on 23-MAY-2015 after a check regarding taxonomy and language by ARVI.

A release date of 01-MAR-2015 of the genome sequence of Alfalfa latent virus (ALV) (GenBank accession KP784454) obviously precedes the submission of our paper to ARVI (13-MAR-2015). It must also be recognized that preparation of a paper requires a significant amount of time, such that the phylogenetic analysis and writing for our paper were done months before the final submission date. The phylogenetic analysis of PeSV was first completed by Li Su on 29-DEC-2014, and finalized on 24-FEB-2015. We could not predict that another related genome sequence would be released in March 2015 and there was no obvious reason to continue to update our analysis in the final weeks before our manuscript submission. This is probably a fairly

This reply refers to the comment available at doi:10.1007/s00705-015-2594-9.

Yu Xiang

yu.xiang@agr.gc.ca

1 Agriculture and Agri-Food Canada, Pacific Agri-Food, Research Centre, Summerland, BC VOH 1Z0, Canada common approach to publication and so we are dismayed that our unintended oversight of the ALV sequence KP784454 was seen as an intentional lack of respect.

There were almost no research updates on either ALV or PeSV over the past thirty years. A comprehensive description of ALV and PeSV can be found in the following websites: http://www.dpwweb.net/dpv/showdpv. php?dpvno=211 (ALV) and http://www.dpwweb.net/dpv/ showdpv.php?dpvno=112 (PeSV).

According to information in the website, "Alfalfa latent virus is serologically unrelated to red clover vein mosaic and pea streak viruses, and it also differs from all other legume carlaviruses in coat protein M. Wt (Veerisetty \& Brakke, 1977b)." (http://www.dpvweb.net/dpv/showdpv. php?dpvno=211). We have discussed and established the molecular relationship between PeSV and RCVMV based on the complete genome sequences of the two viruses in our PeSV paper (Su et al. 2015). Discussing and determining the molecular relationship between PeSV and ALV using their complete genome sequence data would have added value to our paper if the ALV KP784454 accession had been noticed by us in time. The two virus sequences (KP828803 and KP784454) have $77.3 \%$ identity at nucleotide level.

We have made tremendous efforts to respond to Dr. Nemchinov with honesty, respect and understanding, but the communications were extremely frustrating. The heated charges leveled by Dr. Nemchinov appear to lack the respect he is expecting to be applied in the other direction. Our hope is that the scientific community will recognize the need for respect to be mutual in the very human endeavour of research publication.

Sincerely,

Yu Xiang and co-authors 